\title{
Effects of removal of chicks from hens on concentrations of prolactin, luteinizing hormone and oestradiol in plasma of brooding Gifujidori hens
}

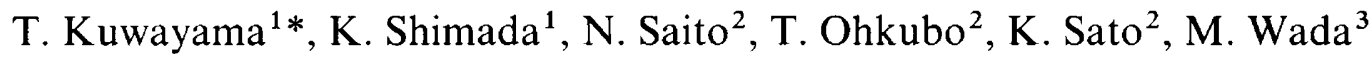 \\ and $\mathrm{K}$. Ichinoe ${ }^{1}$ \\ ${ }^{1}$ Department of Zootechnical Science, Tokyo University of Agriculture, Setagaya-ku, Tokyo 156, \\ Japan; ${ }^{2}$ Department of Animal Physiology, Nagoya University, Chikusa, Nagoya 464-01, Japan; \\ and ${ }^{3}$ Department of General Education, Tokyo Medical and Dental University, Ichikawa,
}

Chiba 272, Japan

\begin{abstract}
Summary. Gifujidori hens were allowed to repeat a breeding cycle in one season. In the first breeding cycle the duration of the brooding (raising chicks) stage was limited to 3 weeks, whereas in the second breeding cycle it was limited to 1 week by removing all chicks from mother hens. In the first breeding cycle, plasma prolactin (PRL) was high during the incubation period, but rapidly decreased on the day of hatching and reached minimum values about 1 week after hatching. In contrast, plasma luteinizing hormone (LH) concentrations were low during the incubation period, but after hatching they gradually increased and reached peak values immediately after removal of chicks. Concentrations of oestradiol in plasma were low in the incubation and brooding stages but increased significantly immediately after removal of chicks. In the second breeding cycle, changes in PRL and LH concentrations were similar to those observed in the first breeding cycle except that even greater increases in plasma LH and oestradiol concentrations were observed one week after hatching when the chicks were removed. These results suggest that coexistence of newly hatched chicks may suppress LH secretion from the pituitary of the hen in the natural breeding cycle.
\end{abstract}

Keywords: prolactin; LH; oestradiol; chicks; hen

\section{Introduction}

The onset of incubation behaviour in chickens and turkeys is characterized by an increase in the concentration of prolactin (PRL) in plasma (Sharp et al., 1979, 1988; Burke \& Dennison, 1980; Lea et al., 1981; Zadworny et al., 1985, 1988; Shimada et al., 1987) and a decrease in the concentrations of luteinizing hormone (LH) in plasma (Cogger et al., 1979; Sharp et al., 1979; Lea et al., 1981) and sex steroid hormones (Cogger et al., 1979; Kono et al., 1985). High concentrations of PRL and low concentrations of $\mathrm{LH}$ and sex steroid hormones in plasma are maintained throughout the incubation period. This inverse relationship between concentrations of PRL and LH in plasma, however, is not applied to the brooding (raising chicks) period after hatching. During the brooding period, plasma LH concentrations remain low (Sharp et al., 1979), whereas those of PRL begin to fall immediately after hatching (Sharp et al., 1979). Plasma LH concentrations usually increase about 3 weeks after hatching when hens take care of chicks. This indicates that newly hatched chicks stimulate maternal behaviour (brooding) which, in turn, suppresses LH secretion and gonadal activity. If the coexistence of chicks with hens is involved in inhibition of release of $\mathrm{LH}$ in

*Reprint requests. 
the hen, it is expected that $\mathrm{LH}$ is released in plasma in the hen in response to removal of chicks from the parent much earlier than 3 weeks after hatching. Sharp et al. (1979) demonstrated that concentration of $\mathrm{LH}$ in plasma increased within 4 days of removing the chicks.

This study investigated the more rapid effects of removal of chicks on the responsiveness of the pituitary-ovarian system by measuring concentrations of LH and oestradiol in plasma of brooding Gifujidori hens at the onset of the second laying cycle.

\title{
Materials and Methods
}

\begin{abstract}
Animals
Four one-year-old Gifujidori hens were housed in individual floor pens $\left(57 \times 57 \times 57 \mathrm{~cm}^{3}\right)$ with nest boxes $\left(41 \times 21 \times 41 \mathrm{~cm}^{3}\right)$. They were maintained in $14 \mathrm{~h}$ light and $10 \mathrm{~h}$ dark (lights on from 05:00 to 19:00 h) with free access to feed and water. Room temperature was maintained at $20 \pm 2^{\circ} \mathrm{C}$. Egg production was recorded daily between 16:00 and 17:00 h. The onset of incubation was defined as the first day when the hens spent more than $90 \%$ of each day on the nest (Lea et al., 1981). When the hens had incubated their eggs in the nests for 16 days, unfertilized eggs were substituted by fertilized eggs that had been incubated in an artificial incubator for 16 days from the onset of incubation. Chicks were hatched during a 1-3 day interval and with brood sizes of between three and ten chicks. Unhatched eggs were removed from the nests 3 days after the first eggs were hatched. The chicks were removed from hens one week (group 1) or 3 weeks (group 2) after hatching.
\end{abstract}

\section{Assays}

Blood samples were collected from a wing vein between 12:00 and 13:00 h every two days. Plasma samples were stored at $-20^{\circ} \mathrm{C}$ until assayed. Plasma concentrations of PRL were measured by the radioimmunoassay method using turkey PRL antigen (Proudman \& Corcoran, 1981) and turkey PRL antibody (Burke \& Papkoff, 1980). Concentrations of LH and oestradiol in plasma were measured by the radioimmunoassay methods of Hattori \& Wakabayashi (1979) for LH and that of Kono et al. (1981) for oestradiol. The intra-assay coefficients for PRL, LH and oestradiol were $15 \cdot 3 \%, 7 \cdot 0 \%$ and $19 \cdot 1 \%$, respectively.

\section{Statistical analysis}

Statistical differences were determined by Duncan's new multiple range test.

\section{Results}

\section{Experiment 1. Effects of removal of chicks from hens 3 weeks after hatching}

Laying began $26 \pm 10$ days after removal of chicks. Concentrations of PRL in plasma were high during the incubation period but rapidly decreased on the day of hatching (i.e. $397.6 \pm 89 \cdot 1 \mathrm{ng} \mathrm{ml}^{-1} 2$ days before hatching; $93.0 \pm 13.7 \mathrm{ng} \mathrm{ml}^{-1}$ on the day of hatching) (Fig. 1). PRL levels dropped further to $17.5 \pm 2.6 \mathrm{ng} \mathrm{ml}^{-1}$ thereafter, but tended to increase in relation to laying. Concentrations of $\mathrm{LH}$ in plasma were low during the incubation period but gradually started to increase during the brooding period.

These fluctuations were not statistically significant from one day to the next but on the basis of stage, the increase in concentrations of $\mathrm{LH}$ in plasma in the pre-laying I stage (between removal of chicks and 10 days after removal) and II stage (between one and 10 days before laying) were significantly different from those in the brooding stage $(P<0.01)$. At time of removal of chicks, LH concentration in plasma markedly increased. This increase was significantly different between one day before and one day after removal $(P<0 \cdot 01)$. The concentration of LH tended to decrease but remained high thereafter. Concentrations of oestradiol remained low both in incubating and brooding stages but significantly increased immediately after removal of chicks $(P<0.01)$ and remained relatively high. 

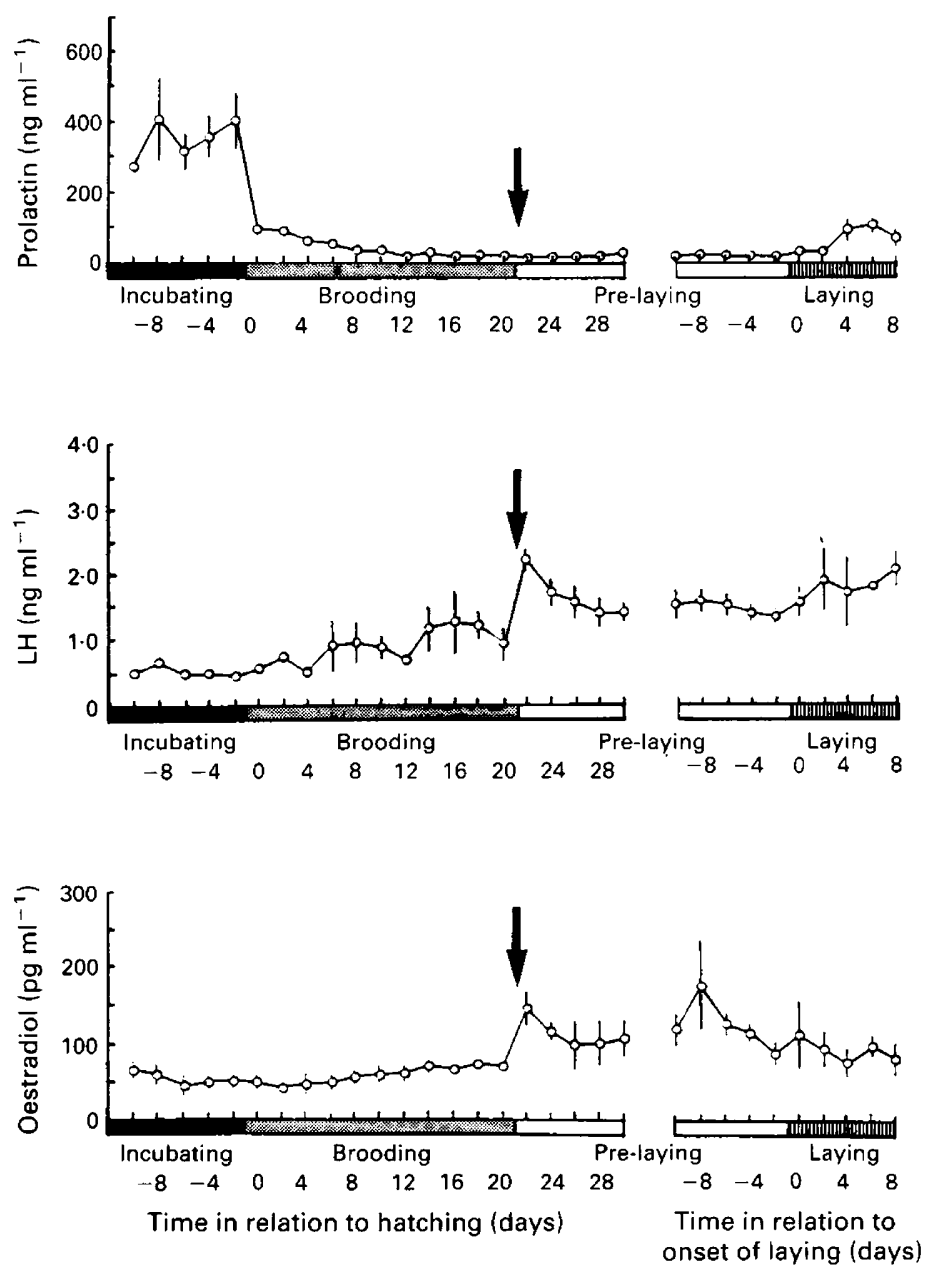

Fig. 1. Concentrations of plasma prolactin, luteinizing hormone $(\mathrm{LH})$ and oestradiol in Gifujidori hens in response to removal of chicks from hens 3 weeks after hatching $(n=4)$. Vertical lines indicate SEM. Arrows indicate removal of chicks.

\section{Experiment 2. Effects of removal of chicks from hens 1 week after hatching}

Laying began $22 \pm 6$ days after chick removal. There was an abrupt fall in concentration of PRL in plasma on the day of hatching of chicks (Fig. 2). The concentration of LH decreased to a minimum value after the removal of chicks. Low concentrations of $\mathbf{L H}$ in plasma were observed both in incubating and brooding stages but the levels sharply increased immediately after removal of chicks. These peak concentrations of $\mathrm{LH}$ were five times higher than the concentrations in the previous stages $(P<0.01)$. The concentrations of LH decreased after several days but the relatively high concentrations were maintained through the successive laying stage. Changes in plasma oestradiol concentrations were similar to those of LH concentrations throughout all the stages. Again, a sharp increase in plasma oestradiol concentrations was noted immediately after removal of chicks $(P<0 \cdot 01)$.

\section{Discussion}

The present study confirmed previous findings of an inverse relationship between plasma concentrations of PRL and LH during the incubation period in hens (Sharp et al., 1979, 1988; Lea et al., 

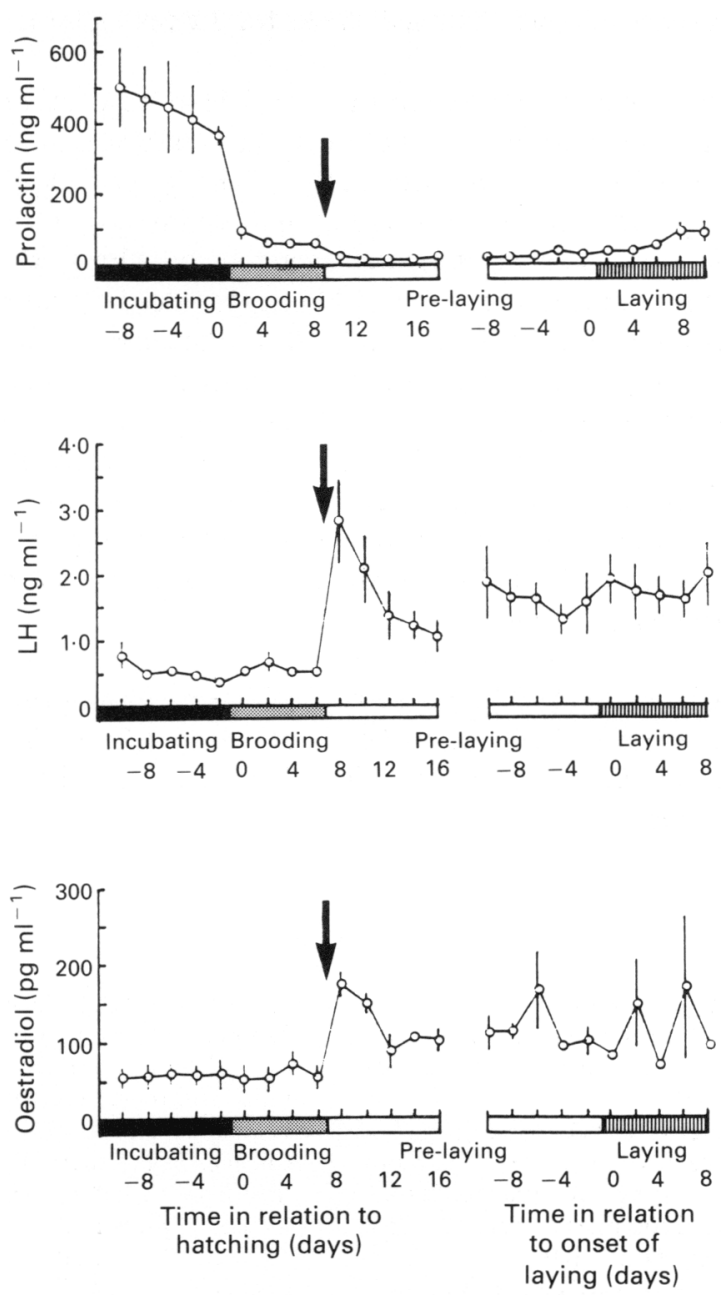

Fig. 2. Concentrations of plasma prolactin, luteinizing hormone (LH) and oestradiol in Gifujidori hens in response to removal of chicks from hens 1 week after hatching $(n=4)$. Vertical lines indicate SEM. Arrows indicate removal of chicks.

1981). However, the underlying mechanisms for the release of these anterior pituitary hormones are not known in hens, but PRL itself may affect the hypothalamo-anterior-pituitary axis and suppress LH release (Sharp et al., 1979, 1988; Lea et al., 1981) or some other factors evoking PRL release (i.e. presence of egg in the nest) may inhibit LH release. Vasoactive intestinal polypeptide (VIP) may be involved in release of PRL in birds (Macnamee et al., 1986; Sharp et al., 1989).

Concentrations of LH in plasma remain low even when plasma PRL concentrations decrease after hatching, suggesting that PRL may not directly inhibit LH release. Furthermore, the present study clearly demonstrated that plasma $\mathrm{LH}$ concentrations rapidly increased at the time chicks were removed from hens. This result indicates that coexistence of chicks with hens is involved in suppression of $\mathrm{LH}$ release in the hen. However, since hens with growing chicks eventually resume egg laying, only newly hatched chicks may provide stimuli for suppressing LH release. These may be auditory (pipping), visual (appearance), physical (tactile) or a combination of these factors. It is uncertain how these stimuli are involved in LH release mechanisms. The possibility that LH release is regulated by internal mechanisms in the hen also cannot be eliminated. Future studies will 
examine whether the presence of newly hatched chicks for 3 weeks after incubation can inhibit LH release in hens.

Finally, it should be noted that mechanisms for $\mathrm{LH}$ release in relation to hatching and broodiness are different from those in hens before sexual maturity, although rising LH concentrations occur in plasma before laying in both cases. In the latter, it has been suggested that the hypothalamo pituitary-gonadal axis is activated by photostimulation as well as by other factors (for example food availability and environmental temperature) which, in turn, results in release of LH during the pre-laying period. In the case of $\mathrm{LH}$ release after broodiness, coexistence of newly hatched chicks involves an inhibitory factor and delays the onset of lay once the hens become sexually mature. During the period of incubation, concentrations of $\mathrm{LH}$ and oestradiol in plasma decrease and yellow yolky follicles in the ovary start regressing. However, the immediate and rapid increase in concentrations of oestradiol in plasma in response to removal of chicks (Figs 1 and 2) suggest that the ovary is steroidogenically active. The primary source of oestrogens is the immature small follicles (Armstrong, 1985; Robinson \& Etches, 1986). In addition to those factors, a modified mechanism for $\mathrm{LH}$ release by coexistence of newly hatched chicks may result in $\mathrm{LH}$ suppression and delay the onset of lay.

We thank J. A. Proudman (USDA, Beltsville, MD) for turkey prolactin, and W. H. Burke (University of Georgia, Athens, GA) for turkey prolactin antibody. This work was supported in part by the Ishida Foundation and a grant-in-aid from the Ministry of Education of Japan (No. 02304027) to K. Shimada.

\section{References}

Armstrong, D.G. (1985) Changes in aromatase activity in small ovarian follicles of the domestic fowl (Gallus domesticus) during growth and atresia. Journal of Endocrinology 105, 297-301.

Burke, W.H. \& Dennison, P.T. (1980) Prolactin and luteinizing hormone levels in female turkey (Meleagris gallopavo) during a photoinduced reproductive cycle and broodiness. General and Comparative Endocrinology 41, 92-100.

Burke, W.H. \& Papkoff, H. (1980) Purification of turkey prolactin and the development of a homologous radioimmunoassay for its measurement. General and Comparative Endocrinology 40, 297-307.

Cogger, E.A., Burke, W.H. \& Ogren, L.A. (1979) Serum luteinizing hormone, progesterone, and estradiol levels in relation to broodiness in the turkey (Meleagris gallapavo). Poultry Science 58, 1355-1360.

Hattori, M. \& Wakabayashi, K. (1979) Isoelectric focusing and gel filtration studies on the heterogeneity of avian pituitary luteinizing hormone. General and Comparative Endocrinology 39, 215-221.

Kono, T., Itoh, H. \& Ichinoe, K. (1981) Measurement of sex steroid hormones in blood plasma and follicle of chicks by radioimmunoassay. Journal of Agricultural Science Tokyo, Nogyo Daigaku, Commemoration Number of 90 th Anniversary of Foundation, 257-265.

Kono, T., Kuwayama, T., Itoh, H. \& Ichinoe, K. (1985) Nesting behavior and changes in plasma concentrations of progesterone, testosterone and estradiol in the native Japanese breed of chicken, Gifujidori (Gallus domesticus). Japanese Poultry Science 22, 64-77.
Lea, R.W., Dods, S.M., Sharp, P.J. \& Chadwick, A. (1981) The possible role of prolactin in the regulation of nesting behaviour and the secretion of luteinizing hormone in broody bantams. Journal of Endocrinology 91, 89-97.

Macnamee, M.C., Sharp, P.J., Lea, R.W., Sterling, R.J. \& Harver, S. (1986) Evidence that vasoactive intestinal polypeptide is a physiological prolactin-releasing factor in the bantam hen. General and Comparative Endocrinology 62, 470-478.

Proudman, J.A. \& Corcoran, D.H. (1981) Turkey prolactin: purification by isotachophoresis and partial characterization. Biology of Reproduction 25, 375-384.

Robinson F.E. \& Etches, R.J. (1986) Ovarian steroidogenesis during follicular maturation in the domestic fowl (Gallus domesticus). Biology of Reproduction 35, 1096-1 105 .

Sharp, P. J., Scanes, C.G., Williams, J.B., Harvey, S. \& Chadwick, A. (1979) Variations in concentrations of prolactin, luteinizing hormone, growth hormone and progesterone in the plasma of broody bantams (Gallus domesticus). Journal of Endocrinology $\mathbf{8 0}$, $51-57$.

Sharp, P.J., Macnamee, M.C., Sterling, R.J., Lea, R.W. \& Pedersen, H.C. (1988) Relationships between prolactin, $\mathbf{L H}$ and broody behaviour in bantam hens. Journal of Endocrinology 118, 279-286.

Sharp, P.J., Sterling, R.J., Talbot, R.T. \& Huskisson, N.S. (1989) The role of hypothalamic vasoactive intestinal polypeptide in the maintenance of prolactin secretion in incubating bantam hens: observations using passive immunization, radioimmunoassay and 
immunohistochemistry. Journal of Endocrinology $122,5-13$.

Shimada, K., Zadworny, D., Ishida, H. \& Sato, K. (1987) Prolactin and estradiol levels in plasma in relation to broodiness in Japanese bantams, (chabo). Kyoto Prolactin Conference Monograph 2, 31-40.

Zadworny, D., Walton, J.S. \& Etches, R.J. (1985) The relationship between plasma concentrations of prolactin and consumption of feed and water during the reproductive cycle of the domestic turkey. Poultry Science 64, 401-410.

Zadworny, D., Shimada, K., Ishida, H., Sumi, C. \& Sato, K. (1988) Changes in plasma levels of prolactin and estradiol, nutrient intake, and time spent nesting during the incubation phase of broodiness in the chabo hen (Japanese bantam). General and Comparative Endocrinology 71, 406-412.

Received 22 May 1991 